\title{
Bevacizumab based chemotherapy is a promising option in metastatic gallbladder adenocarcinoma
}

\author{
SUHAS V. AAGRE ${ }^{1}$, MUBARAKUNNISA TONSE $^{1}$, AVINASH TALELE $^{1}$, \\ SANJAY SHARMA ${ }^{2}$ and SURESH H. ADVANI ${ }^{1}$
}

Departments of ${ }^{1}$ Medical Oncology and ${ }^{2}$ Surgical Oncology, Asian Cancer Institute, Mumbai, Maharashtra 400036, India

Received July 30, 2020; Accepted March 1, 2021

DOI: $10.3892 / \mathrm{mco} .2021 .2315$

\begin{abstract}
Gallbladder cancer (GBC) is one of the most frequently observed cancers in India that is usually diagnosed at an advanced stage. Although surgery remains the only curative option, the majority of GBCs are unresectable. Palliative chemotherapy with gemcitabine and cisplatin is the recommended treatment in such cases. The current study reports a case of a 47-year-old female who exhibited GBC that had metastasized to the liver and peritoneum. She was administered palliative chemotherapy with gemcitabine and cisplatin, but due to disease progression the regimen was changed and an aggressive treatment initiated with gemcitabine and oxaliplatin with additional biosimilar bevacizumab (modified Gemox-B regimen). The patient completed six chemotherapy cycles with partial response and received bevacizumab (7.5 mg/kg 3-weekly) based maintenance treatment for an additional 6 cycles, after which she demonstrated disease progression, thus having a progression free survival of $\sim 11$ months. The patient is currently receiving palliative chemotherapy with capecitabine.
\end{abstract}

\section{Introduction}

Gallbladder cancer (GBC) is the most common biliary tract cancer (BTC) with characteristic thickening of the gallbladder (GB) wall. Most of the GBCs arise from the epithelial lining of the GB and the cystic duct $(1,2)$, and most GBCs are adenocarcinomas (1). The incidence of GBC is higher in the Indian subcontinent than Western counterparts (3). In India, GBC is more common in the northern region when compared with the southern region ( 10-folds) (2).

The only curative option for GBC is surgical resection, however, most of the patients present with unresectable disease at an advanced stage owing to the absence of symptoms in

Correspondence to: Dr Suhas V. Aagre, Department of Medical Oncology, Asian Cancer Institute, 93/95 August Kranti Road, Kemps Corner, Cumballa Hill, Mumbai, Maharashtra 400036, India E-mail: suhas.aagre@gmail.com

Key words: Bevacizumb, biosimilar, gallbladder cancer, biliary tract cancer, Gemox-B the early stages of the disease $(4,5)$. There is no standard chemotherapy established for advanced disease. Palliative chemotherapy with gemcitabine/cisplatin combination or gemcitabine based chemotherapy regimens are recommended for the treatment of metastatic GBC by several treatment guidelines $(3,6)$. Gemcitabine/oxaliplatin (Gemox) combination has shown more potency when compared with gemcitabine/cisplatin combination, and the activity and tolerability of Gemox regimen has been evaluated in cancers, including advanced GBC (7).

Studies have revealed the expression of vascular endothelial growth factor (VEGF) receptor in BTCs and GBCs (8). Addition of an anti-VEGF agent to the chemotherapy could normalize the tumor vasculature and reduce the interstitial pressure in tumors leading to overall improved results. Bevacizumab, an anti-VEGF, is an approved agent for the treatment of metastatic colorectal cancer and has shown effectiveness in several other cancers (9). Bevacizumab in addition to gemcitabine and oxaliplatin chemotherapy (Gemox-B regimen) has shown antitumor activity with tolerable safety in patients with advanced GBCs (8). We report here a case of a patient with GBC adenocarcinoma metastasized to liver and peritoneum who was treated with biosimilar bevacizumab based modified Gemox-B regimen.

\section{Case report}

A 47-year-old woman presented with complaints of right shoulder pain, abdominal pain, decreased appetite and dyspepsia for 6 months. There was no weight loss, fever, comorbidity or any surgical history. Ultrasonography (USG) in July 2019 revealed polypoidal wall thickening of gallbladder with maximum thickness in the fundal region and a mild central intrahepatic biliary radical dilatation suspicious of neoplastic etiology. Bilateral ovaries were bulky (left $>$ right) and hypoechoic. There was moderate left sided hydronephrosis, with upper hydroureter while the distal ureter was obscured by bowel gas.

Fig. 1 provides the details of diagnostic and treatment approaches used in this patient. The computed tomography (CT) showed irregular heterogeneously enhanced eccentric wall thickening involving the gallbladder mainly in the fundal region, loss of fat plane with segment $\mathrm{V}$ of liver and hepatic flexure of colon, focally indistinct fat plane of the D1 segment of duodenum with pericholecystic fat stranding and enhanced circumferential wall of the cystic duct suggestive of GBC. Multiple irregularly shaped hypodense heterogeneously 
enhancing soft tissue nodules were seen in the pericholecystic region and serosal surface of the ascending colon, and caecum/small soft tissue density nodule in the anterior abdominal wall in the umbilical region. Also, multiple small hypodense nodules scattered in both lobes of liver mainly in segment VIII, IVb and III, were seen. These findings were suggestive of metastatic disease. Mild ascites was present.

The histological examination revealed few scattered clusters of medium sized ductal cells exhibiting pleomorphic, irregular and mildly overlapping dark staining nuclei (Fig. 2).

The baseline positron emission tomography with 2-deoxy-2-[fluorine-18]fluoro-D-glucose integrated with computed tomography $\left({ }^{18} \mathrm{~F}-\mathrm{FDG}\right.$ PET-CT) done on 11-Jul-2019 revealed asymmetric FDG avid enhancing thickening of wall of the distal body and fundic region of the GBC (soft tissue mass measuring $3.4 \times 2.8 \times 2.9 \mathrm{~cm}$; standardized uptake value $\left.\left[\mathrm{SUV}_{\max }\right]: 6.08\right)$, and confirmed the peritoneal and liver metastasis with narrowing of the common bile duct (CBD). Bulky bilateral ovaries (left ovary: 3.8x3.0x2.9; left more than right with avid FDG uptake) with left sided hydronephrosis and hydroureter were seen $\left(\mathrm{SUV}_{\max }: 5.35\right)$.

Her laboratory investigations were abnormal-CA-125: 117.2 U/ml (normal: <46 U/ml), CEA: $5.5 \mathrm{ng} / \mathrm{ml}$ (normal: $<5 \mathrm{ng} / \mathrm{ml}$ ), CA19.9: $1433.0 \mathrm{U} / \mathrm{ml}$ (normal: $<37 \mathrm{U} / \mathrm{ml}$ ), creatinine: $1.3 \mathrm{mg} / \mathrm{dl}$ (normal: $0.84-1.21 \mathrm{mg} / \mathrm{dl}$ ), total bilirubin: $5.5 \mathrm{mg} / \mathrm{dl}$ (normal: $0.3 \mathrm{mg} / \mathrm{dl}$ ), alanine transaminase (ALT): $356 \mathrm{U} / 1$ (normal: $<40 \mathrm{U} / \mathrm{l}$ ), aspartate transaminase (AST): $95 \mathrm{U} / 1$ (normal: <40 U/l), alkaline phosphatase (ALP): $421 \mathrm{U} / 1$ (normal: 20-140 IU/l), gamma-glutamyl transferase (GGT): $187 \mathrm{U} / 1$ (normal: <30 IU/l). Her total proteins were $7.84 \mathrm{~g} / \mathrm{dl}$ (normal: 6-8.3 g/dl), albumin $4.02 \mathrm{~g} / \mathrm{dl}$ (normal: 3.4-5.4 g/dl) and globulin $3.82 \mathrm{~g} / \mathrm{dl}$ (normal: 2-3.5 g/dl).

Magnetic resonance cholangiopancreatography (MRCP) performed on 16-Jul-2019 suggested neoplastic lesion involving fundus of the gallbladder infiltrating adjacent liver parenchyma. She underwent endoscopic retrograde cholangiopancreatography (ERCP) with CBD stenting on 18-Jul-2019. Biliary stricture was seen and self-expandable metallic stent (SEMS; 10x80 mm) was placed for Bismuth type II biliary stricture with cholangitis.

Her liver function tests gradually improved over one week The cancer staging was evaluated as $\mathrm{T}_{3} \mathrm{~N}_{2} \mathrm{M}_{1}$ - Stage IV. The patient was planned to receive three chemotherapy cycles with intravenous (IV) gemcitabine $1,000 \mathrm{mg} / \mathrm{m}^{2}$ and cisplatin $25 \mathrm{mg} / \mathrm{m}^{2}$ on Days 1 and 8 every 21 days. She received the $1^{\text {st }}$ dose of cycle 1 chemotherapy on 6-Aug-2019 (Day 1) and the second dose on 13-Aug-2019 (Day 8).

After 4-5 days of the first chemotherapy dosing, she complained of vomiting (green colored), abdominal pain and generalized weakness (all grade 1-2), which improved with conservative treatment. On multidetector CT (MDCT) performed on 22-Aug-2019, few heterogeneously enhancing lesions were seen in the liver (largest: $13 \mathrm{~mm}$, Segment IV-a). A large heterogeneously enhancing centrally necrotic mass lesion in the gastrosplenic region, encasing the tail of pancreas suggestive of large metastatic deposits $(7.5 \times 4.2 \times 8.7)$ was a new finding. There was a mild interval increase in the size of bilateral ovarian lesions. These aforementioned MDCT findings were suggestive of disease progression, and hence, the regimen was changed to an aggressive approach with triple drug combination (modified Gemox with bevacizumab) of gemcitabine $900 \mathrm{mg} / \mathrm{m}^{2}$, oxaliplatin $80 \mathrm{mg} / \mathrm{m}^{2}$ (Days 1 and 8 in each 3-weekly cycle) and bevacizumab $7.5 \mathrm{mg} / \mathrm{Kg}$ (on Day 1 in each 3-weekly cycle). Her symptoms gradually improved with the treatment. The modified Gemox-B regimen was well-tolerated with no significant abnormal laboratory investigations. In view of clinical response, patient was treated with four cycles of bevacizumab based chemotherapy.

Interim PET-CT showed reduction in lesions all over except in the peritoneum; liver lesions were resolved. The baseline (Fig. 3A) and interim PET-CT images are shown (Fig. 3B).

The wall of gallbladder was irregularly thickened, which was significantly reduced in size and metabolic activity $\left(\mathrm{SUV}_{\max }\right.$ was 2.45 ; largest measuring size: $10 \mathrm{~mm}$ ). The previously noted left ovarian lesions showed reduction in size $\left(3 \times 2.5 \mathrm{~cm} ; \mathrm{SUV}_{\max }: 1.88\right)$. Subsequently, the patient was administered fifth and sixth chemotherapy cycles.

The ${ }^{18}$ F-FDG PET-CT after six chemotherapy cycles showed interval reduction in metabolic activity of ill-defined soft tissue lesions involving fundus of gallbladder. The $\mathrm{SUV}_{\max }$ was 1.77 and measured approximately $1.5 \times 1 \mathrm{~cm}$. Interval reduction in the size of non-FDG avid serosal deposits in subhepatic space was seen, and low grade metabolically active bilateral ovarian lesions were unchanged; left ovarian lesion with $\mathrm{SUV}_{\max } 1.44$ measuring $\sim 3.1 \times 2.1 \mathrm{~cm}$ and right ovarian lesion with $\mathrm{SUV}_{\max }$ 1.72 measuring $\sim 2.1 \times 1.6 \mathrm{~cm}$. There was interval reduction in the number of non-FDG avid liver lesions. No other evidence of metabolically active disease was seen (Fig. 4).

Her CA19-9 levels decreased to $70 \mathrm{U} / \mathrm{ml}$. Further maintenance treatment with bevacizumab $(7.5 \mathrm{mg} / \mathrm{kg} 3$-weekly) was planned in view of the excellent response. The patient tolerated the maintenance treatment well without any adverse events (AEs). Unfortunately, she developed clinically progressive disease with reappearance of symptoms like right sided abdominal pain, nausea and loss of appetite in June 2020 (progression free interval: 11 months). PET-CT done in June 2020 suggested persistent primary gallbladder lesion with mild increase in the number and metabolic activity of the serosal deposits in the subhepatic space. Mild increases in the size and metabolic activity were noted in the bilateral ovarian lesions. Currently, the patient has been started on palliative chemotherapy with capecitabine.

\section{Discussion}

Gallbladder cancer is a very aggressive and difficult to treat BTC (10). Studies have reported 6-9 months progression free survival and 9.8-14 months overall survival in advanced unresected GBC patients (11). Surgery remains the only curative option but only $10 \%$ patients are eligible as majority of the cases are presented at an advanced disease state $(6,12)$. Most GBCs do not respond well to chemotherapy with a single agent (13). For locally advanced or metastatic unresectable GBC, gemcitabine and cisplatin based chemotherapy is the recommended treatment option $(3,14)$. Furthermore, chemotherapy in combination with bevacizumab has shown promising results in the treatment of BTCs (15). We report here a case of a 47-year-old woman with GBC metastasized to liver and peritoneum who was treated with a biosimilar bevacizumab based Gemox-B regimen. The patient underwent ERCP with CBD stenting following which she received the first chemotherapy cycle with gemcitabine and 

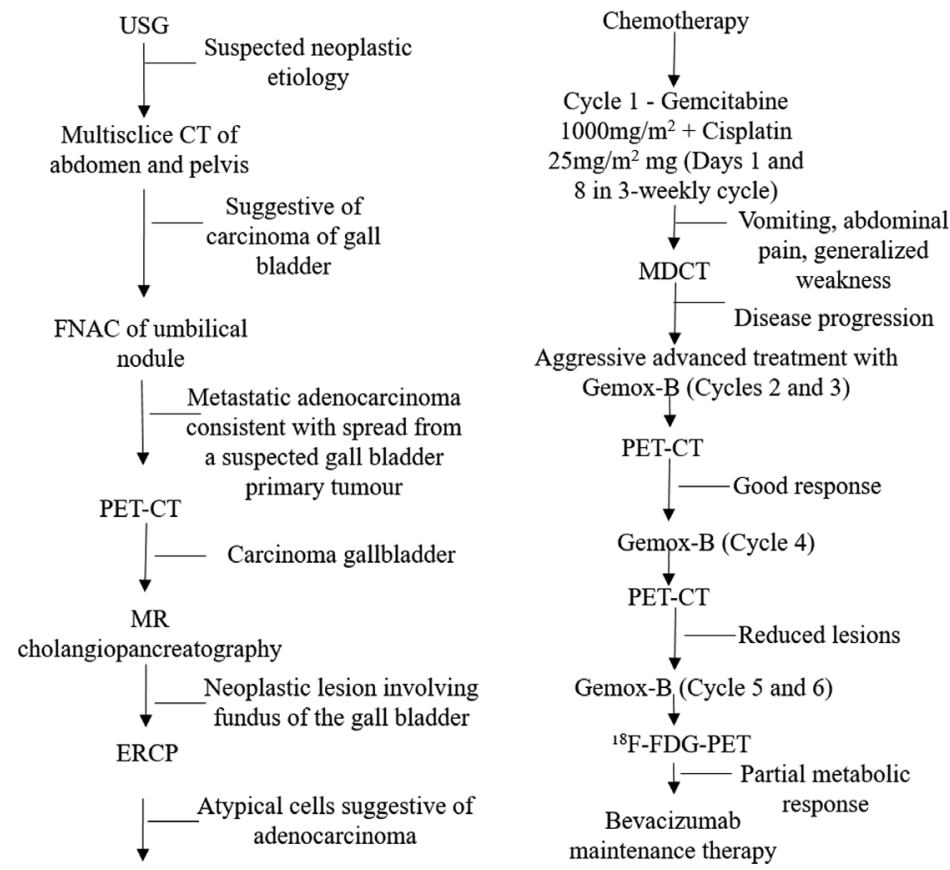

Figure 1. Graphical description of imaging and treatment modalities in a patient with GBC. ${ }^{18}$ F-FDG, 2-deoxy-2-[fluorine-18]fluoro-D-glucose; CT, computed tomography; ERCP, endoscopic retrograde cholangiopancreatography; FNAC, fine needle aspiration cytology; MDCT, multidetector computed tomography; MR, magnetic resonance; PET, positron emission tomography; USG, ultrasonography.

cisplatin. The MDCT performed after the first chemotherapy cycle demonstrated a new finding of a large necrotic mass lesion encasing the pancreatic tail suggesting large metastatic deposits (7.5x4.2x8.7). Such a large mass could not be attributed to the post ERCP pancreatitis, which was confirmed by an independent radiologist and indicated disease progression. Hence, the patient was switched to an aggressive bevacizumab based Gemox-B regimen.

Several factors including advanced age, female gender and Indian origin are among the established risk factors (12), and abdominal pain, discomfort, jaundice, vomiting, abdominal mass and ascites are common clinical manifestations of GBC (1), which were also seen in our patient.

Majority of the GBCs arise in the fundus $(60 \%)$, infiltrate directly in the liver, and spread to different segments (IV and V) of liver and peritoneum, and are of adenocarcinoma (98\%) histology $(12,16,17)$, similar to as seen in our patient. A decrease in pretreatment CA 19-9 levels after chemotherapy are of prognostic relevance in patients with BTCs $(12,18)$. In our patient, the CA19.9 levels were decreased from $1433 \mathrm{U} / \mathrm{ml}$ at baseline to $70 \mathrm{U} / \mathrm{ml}$ post treatment.

The NCCN guidelines suggest investigation with MDCT or contrast-enhanced MRI with MRCP and chest CT in case of suspicious GBC. Similar imaging modalities were carried-out in our patient. The treatment guidelines suggest gemcitabineor fluoropyrimidine-based chemotherapy for the treatment of advanced unresectable GBCs $(3,6)$. Gemcitabine has shown clinical benefit rates (complete response+partial response+stable disease) of $15-60 \%$ in GBC cases (12). The combination of gemcitabine and cisplatin when compared with gemcitabine alone has resulted in increased response rates (26 vs. 16\%) and OS (11.7 vs. 8.1 months) in GBC (19). A systematic review of clinical studies show that gemcitabine and oxaliplatin combination (Gemox) regimen has a better toxicity profile without significant difference in the efficacy compared with cisplatin/gemcitabine regimen (20). Furthermore, Gemox regimen has demonstrated a response rate of 26 to $50 \%$ and an OS of 11 to 15.4 months in the treatment of advanced gallbladder and BTCs (12).

The combination of molecularly targeted agents with chemotherapy have also been evaluated in patients with BTCs with promising efficacy and tolerability (21-23). Letelier et al, have reported that VEGF-A are expressed in 81\% (183/224) of GBC cases (24). These findings suggest a possible role of anti-VEGF agent for the treatment of GBCs. Bevacizumab, an anti-VEGF, has been successfully combined with chemotherapy for several cancers including GBC (8). The Gemox-B regimen has reported promising results (response rate: 40\%, median OS: 12.7 months) in the treatment of GBCs in a phase II study (8). Innovator biologic bevacizumab based Gemox-B regimen has been used previously in the GBCs. In our patient, a biosimilar bevacizumab (Bevatas of Intas Pharmaceuticals Limited, India) based modified Gemox-B regimen was used, which demonstrated a partial response as per RECIST 1.1 and a partial metabolic response (PMR). Intas' biosimilar bevacizumab was approved in India in 2017, with an intent to provide a cost-effective (up to $40 \%$ ) alternative formulation of innovator bevacizumab to the Indian patients (25).

The FDG combined with PET scan is an early, sensitive, pharmacodynamic marker of the tumoricidal effect of chemotherapeutic agents. In our patient, ${ }^{18} \mathrm{~F}-\mathrm{FDG}-\mathrm{PET}$ analysis showed a mean decrease in $\mathrm{SUV}_{\max }$ of $\sim 70 \%\left(\mathrm{SUV}_{\max }\right.$-baseline: 6.08; post-treatment 1.77; difference: 4.31) suggesting a PMR as per EORTC criteria (26). Previous studies have correlated that a significantly larger decrease in $\mathrm{SUV}_{\max }$ was observed in patients with longer PFS (>6 months) and overall survival (OS, $>12$ months) (26).

The reported tumor response rate (complete response rate + partial response rate + stable disease) in the phase II study with Gemox-B regimen was $69 \%$. However, complete response was not seen in any of the patients (out of 35) (8). In our patient, a 


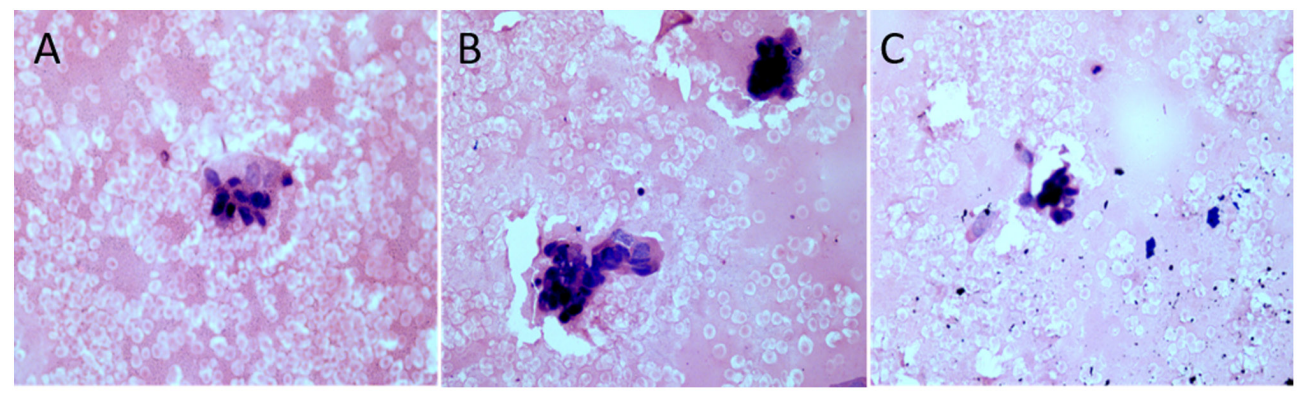

Figure 2. Histological examination showing metastatic gallbladder adenocarcinoma. (A) high power view (magnification, $\mathrm{x} 40$ ) of a tumour giant cell. (B) High power view (magnification, $\mathrm{x} 40$ ) of adenocarcinoma cell clusters. (C) Low power view (magnification, x10) of clusters and a few individually dispersed adenocarcinoma cells.
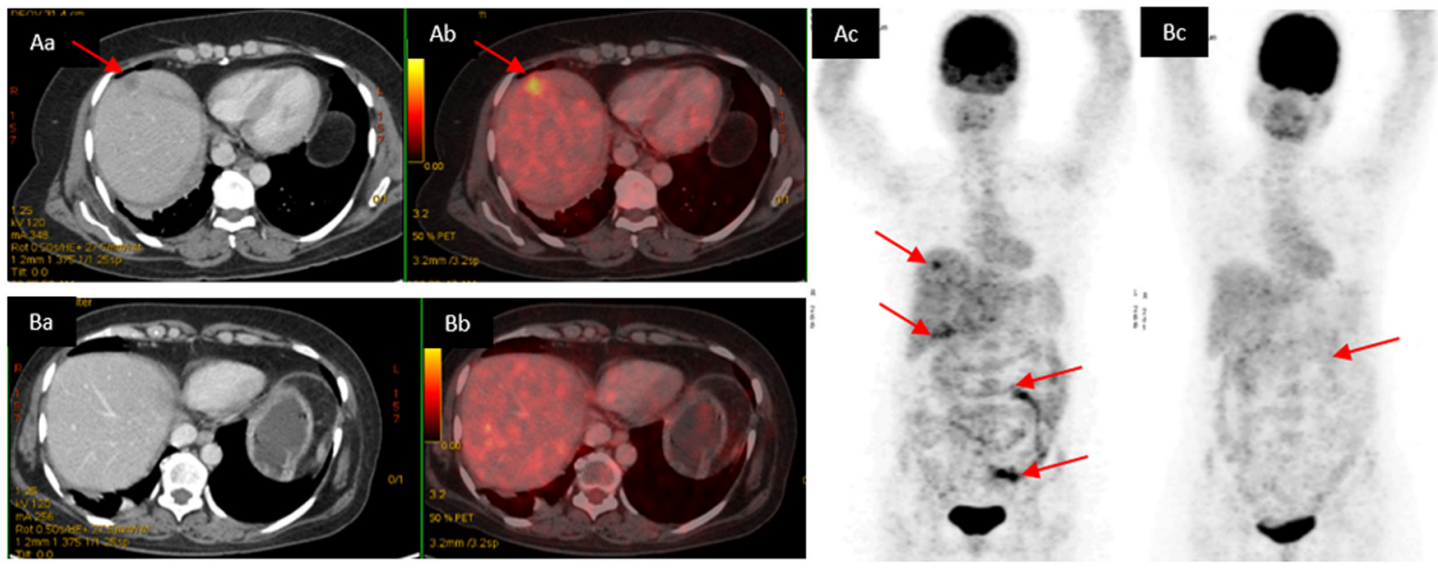

Figure 3. Baseline images showing hypermetabolic thickening of the gallbladder, along with peritoneal and liver metastasis with bulky bilateral ovaries. (Aa) CT, (Ab) ${ }^{18} \mathrm{~F}-\mathrm{FDG}$ PET-CT and (Ac) PET scan images are presented. Interim (Ba) CT scan, (Bb) ${ }^{18} \mathrm{~F}-\mathrm{FDG}$ PET-CT and (Bc) PET scan images post four treatment cycles showing a reduction in lesions, except in the peritoneum.
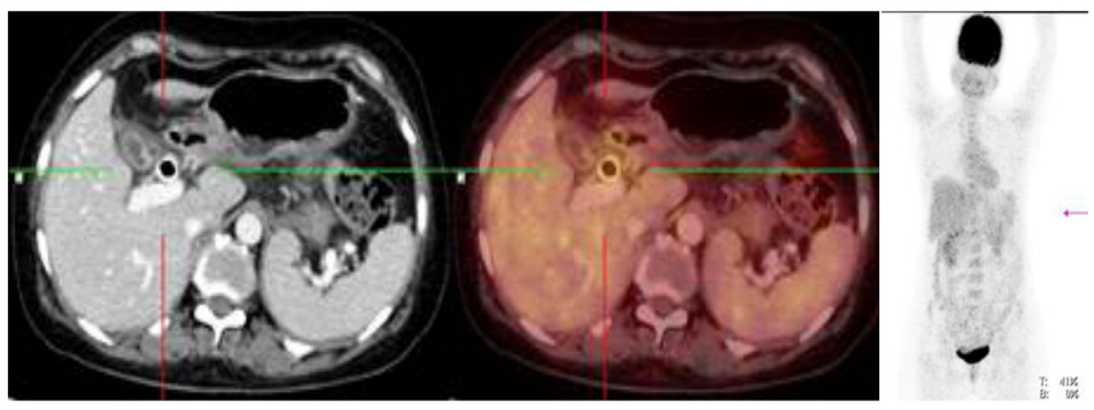

Figure 4. Post treatment ${ }^{18} \mathrm{~F}$-FDG PET-CT showing interval reduction in metabolic activity of ill-defined soft tissue lesions involving the fundus of the gallbladder and non-FDG avid liver lesions.

partial response was observed after Gemox-B chemotherapy. At 6 months follow-up post bevacizumab based chemotherapy, the patient developed disease progression showing a progression-free survival of $\sim 11$ months, which is comparatively greater than those reported in the literature (11). The patient is still alive and currently receiving palliative chemotherapy with capecitabine. In our patient, there was no grade III or IV AEs observed which is in line with the results from the phase II study where Gemox-B regimen showed a favorable tolerability profile in patients with BTCs (8).

Overall, biosimilar bevacizumab based modified Gemox-B regimen resulted in a partial response and a progression free survival of $\sim 11$ months in a patient with advanced gallbladder cancer. The current case could provide insights for biosimilar bevacizumab based chemotherapy regimen options for the palliative treatment of metastatic gallbladder carcinoma and need to be further evaluated in clinical studies.

\section{Acknowledgements}

The authors would like to thank Dr Jaykumar Sejpal and Dr Mujtaba A. Khan (both Intas Pharmaceuticals Ltd.) for their medical review of the manuscript. The authors would also like to thank Mr. Shreekant Sharma (ISMPP CMPPтм; 
Intas Pharmaceuticals Ltd.) for his support in developing the concept/medical writing, additional editorial support and follow-up with the journal. Finally, the authors acknowledge Dr Venugopal Madhusudhana (ISMPP CMPPтм; Intas Pharmaceuticals Ltd.) for additional editorial assistance.

\section{Funding}

No funding was received.

\section{Availability of data and materials}

The datasets used and/or analyzed during the current study are available from the corresponding author on reasonable request.

\section{Authors' contributions}

SVA, MT, AT, SS and SHA participated in study design, data collection, data interpretation, and writing/review of the case report. All authors substantially contributed to interpretation of data, critical revision of manuscript, and consented to the final version of the case report. SVA and SHA confirm the authenticity of all the raw data. All authors read and approved the final manuscript.

\section{Ethics approval and consent to participate}

Not applicable.

\section{Patient consent for publication}

A signed informed consent document was obtained from the patient for the publication of this case report and associated data.

\section{Competing interests}

The authors declare that they have no competing interests.

\section{References}

1. Maplanka C: Gallbladder cancer, treatment failure and relapses: The peritoneum in gallbladder cancer. J Gastrointest Cancer 45: 245-255, 2014.

2. Dutta U, Bush N, Kalsi D, Popli P and Kapoor VK: Epidemiology of gallbladder cancer in India. Chin Clin Oncol 8: 33, 2019.

3. Valle JW, Borbath I, Khan SA, Huguet F, Gruenberger T and Arnold D; ESMO Guidelines Committee: Biliary cancer: ESMO Clinical Practice Guidelines for diagnosis, treatment and follow-up. Ann Oncol 27 (Suppl 5): v28-v37, 2016

4. Wang H, Ling W and Luo Y: Contrast-enhanced ultrasound findings of gallbladder adenocarcinoma with sarcomatoid carcinoma accompanied by intrahepatic metastasis: A case report and literature review. Medicine (Baltimore) 97: e10773, 2018.

5. Oh J, Steel M, Conklin C and Aquino-Parsons C: Metastatic gallbladder adenocarcinoma to the endometrium: A case report and review of literature. Cureus 11: e5258, 2019.

6. National Comprehensive Cancer Network. Hepatobilliary Cancers (Version 2.2019) . (2019). Accessed: Jannuary 31, 2020 : https://www.nccn.org/professionals/physician_gls/PDF/hepatobiliary.pdf.

7. André T, Tournigand $\mathrm{C}$, Rosmorduc O, Provent $\mathrm{S}$, Maindrault-Goebel F, Avenin D, Selle F, Paye F, Hannoun L, Houry S, et al: Gemcitabine combined with oxaliplatin (GEMOX) in advanced biliary tract adenocarcinoma: A GERCOR study. Ann Oncol 15: 1339-1343, 2004.
8. Zhu AX, Meyerhardt JA, Blaszkowsky LS, Kambadakone AR, Muzikansky A, Zheng H, Clark JW, Abrams TA, Chan JA, Enzinger PC, et al: Efficacy and safety of gemcitabine, oxaliplatin, and bevacizumab in advanced biliary-tract cancers and correlation of changes in 18-fluorodeoxyglucose PET with clinical outcome: A phase 2 study. Lancet Oncol 11: 48-54, 2010.

9. Keating GM: Bevacizumab: A review of its use in advanced cancer. Drugs 74: 1891-1925, 2014.

10. Uji M, Mizuno T, Ebata T, Sugawara G, Igami T, Uehara K and Nagino M: A case of advanced intrahepatic cholangiocarcinoma accidentally, but successfully, treated with capecitabine plus oxaliplatin (CAPOX) therapy combined with bevacizumab: A case report. Surg Case Rep 2: 63, 2016.

11. Sacchetti F, Ardito F, Vecchio FM and Giuliante F: Exceptional long-term survivor (12 years) with metastatic gallbladder cancer. Glob Surg 5, 2019.

12. Kanthan R, Senger JL, Ahmed S and Kanthan SC: Gallbladder cancer in the 21st century. J Oncol 2015: 9674722015.

13. Caldow Pilgrim CH, Groeschl RT, Quebbeman EJ and Gamblin TC: Recent advances in systemic therapies and radiotherapy for gallbladder cancer. Surg Oncol 22: 61-67, 2013.

14. Athauda A, Fong C, Lau DK, Javle M, Abou-Alfa GK, Morizane C, Steward K and Chau I: Broadening the therapeutic horizon of advanced biliary tract cancer through molecular characterisation. Cancer Treat Rev 86: 101998, 2020.

15. Zhang W, Zhou H, Wang Y, Zhang Z, Cao G, Song T, Zhang T and Li Q: Systemic treatment of advanced or recurrent biliary tract cancer. Biosci Trends 14: 328-341, 2020.

16. Lim KS, Peters CC, Kow A and Tan CH: The varying faces of gall bladder carcinoma: Pictorial essay. Acta Radiol 53: 494-500, 2012.

17. Yoshimitsu K, Nishihara Y, Okamoto D, Ushijima Y, Nishie A, Yamaguchi K, Taketomi A and Honda $\mathrm{H}$ : Magnetic resonance differentiation between T2 and T1 gallbladder carcinoma: Significance of subserosal enhancement on the delayed phase dynamic study. Magn Reson Imaging 30: 854-859, 2012.

18. Harder J, Kummer O, Olschewski M, Otto F, Blum HE and Opitz O: Prognostic relevance of carbohydrate antigen 19-9 levels in patients with advanced biliary tract cancer. Cancer Epidemiol Biomarkers Prev 16: 2097-2100, 2007.

19. Valle J, Wasan H, Palmer DH, Cunningham D, Anthoney A, Maraveyas A, Madhusudan S, Iveson T, Hughes S, Pereira SP, et al: Cisplatin plus gemcitabine versus gemcitabine for biliary tract cancer. N Engl J Med 362: 1273-1281, 2010.

20. Fiteni F, Nguyen T, Vernerey D, Paillard MJ, Kim S, Demarchi M, Fein F, Borg C, Bonnetain F and Pivot X: Cisplatin/gemcitabine or oxaliplatin/gemcitabine in the treatment of advanced biliary tract cancer: A systematic review. Cancer Med 3: 1502-1511, 2014.

21. Malka D, Fartoux L, Rousseau V, Trarbach T, Boucher E, De La Fouchardiere C, Faivre SJ, Viret F, Blanc JF, Assenat E, et al: Gemcitabine and oxaliplatin (GEMOX) alone or in combination with cetuximab as first-line treatment for advanced biliary cancer: Final analysis of a randomized phase II trial (BINGO). J Clin Oncol 30: 4032, 2012.

22. Gruenberger B, Schueller J, Heubrandtner U, Wrba F, Tamandl D, Kaczirek K, Roka R, Freimann-Pircher S and Gruenberger T: Cetuximab, gemcitabine, and oxaliplatin in patients with unresectable advanced or metastatic biliary tract cancer: A phase 2 study. Lancet Oncol 11: 1142-1148, 2010.

23. Safran H, Miner T, Resnick M, Dipetrillo T, McNulty B, Evans D, Joseph P, Plette A, Millis R, Sears D, et al: Lapatinib/gemcitabine and lapatinib/gemcitabine/oxaliplatin: A phase I study for advanced pancreaticobiliary cancer. Am J Clin Oncol 31: 140-144, 2008.

24. Letelier P, Garcia P, Leal P, Ili C, Buchegger K, Riquelme I, Sandoval A, Tapia O and Roa JC: Immunohistochemical expression of vascular endothelial growth factor A in advanced gallbladder carcinoma. Appl Immunohistochem Mol Morphol 22: 530-536, 2014.

25. Intas Launches Bevatas (bevacizumab) in India. [cited 09 Nov 2020]. Available from https://www. bigmoleculewatch. com/2017/10/17/intas-launches-bevatas-bevacizumab-india/?highlight= bevatas.

26. Young H, Baum R, Cremerius U, Herholz K, Hoekstra O, Lammertsma AA, Pruim J and Price P: Measurement of clinical and subclinical tumour response using $\left[{ }^{18} \mathrm{~F}\right]$-fluorodeoxyglucose and positron emission tomography: Review and 1999 EORTC recommendations. European Organization for Research and Treatment of Cancer (EORTC) PET Study Group. Eur J Cancer 35: 1773-1782, 1999. 